\title{
The outer disk of the classical Be star $\psi$ Per
}

\section{Robert Klement $^{1,2}$, Alex C. Carciofi ${ }^{3}$, Thomas Rivinius ${ }^{2}$, Lynn D.} Matthews $^{4}$, Richard Ignace ${ }^{5}$, Jon E. Bjorkman ${ }^{6}$, Rodrigo G. Vieira ${ }^{3}$, Bruno C. Mota ${ }^{3}$, Daniel M. Faes ${ }^{3}$, Stanislav Stef ${ }^{\dagger}$

${ }^{1}$ Astronomical Institute of Charles University, Charles University, V Holešovičkách 2, 180 00, Prague 8, Czech Republic, email: robertklement@gmail.com

${ }^{2}$ European Southern Observatory, Alonso de Córdova 3107, Vitacura, Casilla 19001, Santiago, Chile

${ }^{3}$ Instituto de Astronomia, Geofíisica e Ciências Atmosféricas, Universidade de São Paulo, Rua do Matão 1226, 05508-090, São Paulo, Brazil

${ }^{4}$ MIT Haystack Observatory, off Route 40, Westford MA 01886, USA

${ }^{5}$ Department of Physics \& Astronomy, East Tennessee State University, Johnson City, TN 37614, USA

${ }^{6}$ Ritter Observatory, Department of Physics \& Astronomy, University of Toledo, Toledo, OH 43606, USA

${ }^{\dagger}$ Deceased

\begin{abstract}
To this date $\psi$ Per is the only classical Be star that was angularly resolved in radio (by the VLA at $\lambda=2 \mathrm{~cm}$ ). Gaussian fit to the azimuthally averaged visibility data indicates a disk size $(F W H M)$ of $\sim 500$ stellar radii (Dougherty \& Taylor 1992). Recently, we obtained new multi-band cm flux density measurements of $\psi$ Per from the enhanced VLA. We modeled the observed spectral energy distribution (SED) covering the interval from ultraviolet to radio using the Monte Carlo radiative transfer code HDUST (Carciofi \& Bjorkman 2006). An SED turndown, that occurs between far-IR and radio wavelengths, is explained by a truncated viscous decretion disk (VDD), although the shallow slope of the radio SED suggests that the disk is not simply cut off, as is assumed in our model. The best-fit size of a truncated disk derived from the modeling of the radio SED is $100_{-15}^{+5}$ stellar radii, which is in striking contrast with the result of Dougherty \& Taylor (1992). The reasons for this discrepancy are under investigation.
\end{abstract}

Keywords. stars: individual ( $\psi$ Per), stars: emission-line, Be, radio continuum: stars

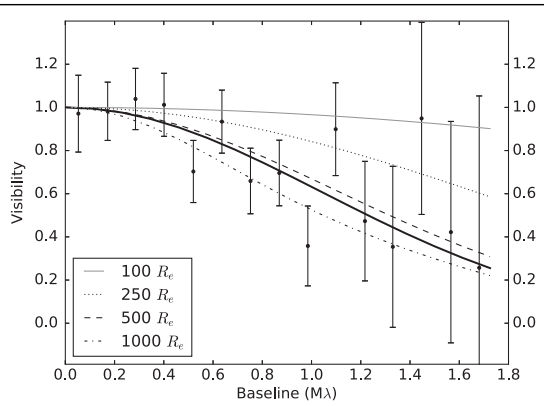

Figure 1. Azimuthally averaged visibility data of Dougherty \& Taylor (points) and a Gaussian fit to them (thick line) overplotted with the visibility curves derived from our azimuthally averaged models with different disk sizes (in stellar equatorial radii $R_{\mathrm{e}}$ ). The disk size best reproducing the radio SED is $100 R_{\mathrm{e}}$, which does not agree well with the interferometric data.

\section{References}

Carciofi, A.C. \& Bjorkman, J.E. 2006, ApJ, 639, 1081

Dougherty, S.M. \& Taylor A.R. 1992, Nature, 359, 808 